Tropical Journal of Pharmaceutical Research February 2019; 18 (2): 397-402

ISSN: $1596-5996$ (print); 1596-9827 (electronic)

(C) Pharmacotherapy Group, Faculty of Pharmacy, University of Benin, Benin City, 300001 Nigeria.

\title{
Combined effect of celecoxib and glucosamine sulfate on inflammatory factors and oxidative stress indicators in patients with knee osteoarthritis
}

\author{
Deng Gang, Chen Xiaguang, Yang Kanghua, Wu Aiping, Zeng Guangxuan* \\ Department of Sports Medicine, People's Hospital of Ganzhou City, Jiangxi Province 341000, China
}

${ }^{*}$ For correspondence: Email: fe1293@163.com

Sent for review: 13 November 2018

Revised accepted: 28 January 2019

\begin{abstract}
Purpose: To investigate the combined effect of celecoxib and glucosamine sulfate on inflammatory factors and oxidative stress indicators in patients with knee osteoarthritis (KOA).

Methods: Patients were randomly assigned to two groups of 60 patients each: control group and study group. The control group received celecoxib at a dose of $200 \mathrm{mg} / \mathrm{kg} /$ day, while the study group received glucosamine sulfate $(500 \mathrm{mg} / \mathrm{kg})$ in addition to celecoxib, thrice a day. Treatment in both groups lasted 8 weeks. The serum levels of tumor necrosis factor a (TNF- $\alpha$ ), interleukin-1 (IL-1), prostaglandin-2 (PGE2), malondialdehyde (MDA), and activity of superoxide dismutase (SOD) were assayed before and after treatment. Visual analogue scale (VAS), osteoarthritis index, Lysholm knee score scale (LKSS), and adverse reactions were also evaluated.

Results: After treatment, total effectiveness was significantly higher in the study group (91.33\%) than in control group (71.67\%, $p<0.05$ ). Serum TNF- $\alpha, I L-1$ and PGE2 were significantly lesser in the glucosamine sulfate-treated patients than in control group $(p<0.05)$. The activity of SOD was significantly higher in glucosamine sulfate-exposed patients than control patients $(p<0.05)$. On the other hand, VAS and WOMAC scores were markedly lower in patients given glucosamine sulfate than in control patients $(p<0.05)$.

Conclusion: The combination of celecoxib with glucosamine sulfate effectively reduces immune inflammatory response, oxidative stress damage, and joint pain associated with KOA.
\end{abstract}

Keywords: Celecoxib, Glucosamine sulfate, Osteoarthritis, Inflammatory factors, Oxidative stress

\begin{abstract}
This is an Open Access article that uses a funding model which does not charge readers or their institutions for access and distributed under the terms of the Creative Commons Attribution License (http://creativecommons.org/licenses/by/4.0) and the Budapest Open Access Initiative (http://www.budapestopenaccessinitiative.org/read), which permit unrestricted use, distribution, and reproduction in any medium, provided the original work is properly credited.

Tropical Journal of Pharmaceutical Research is indexed by Science Citation Index (SciSearch), Scopus, International Pharmaceutical Abstract, Chemical Abstracts, Embase, Index Copernicus, EBSCO, African Index Medicus, JournalSeek, Journal Citation Reports/Science Edition, Directory of Open Access Journals (DOAJ), African Journal Online, Bioline International, Open-J-Gate and Pharmacy Abstracts
\end{abstract}

\section{INTRODUCTION}

Osteoarthritis $(\mathrm{OA})$ is a degenerative disease of bones and joints which occurs mainly in the knee joint, hip joint, spine and other parts in middleaged and elderly people. It is caused by pain and joint dysfunction. Pain of long duration induces local inflammation of joint soft tissue, aggravates damage to cartilage, and seriously reduces quality of life of sufferers [1]. At present, surgery, conservative treatment, and use of antiosteoarthritic drugs are the major treatment options for OA.

Celecoxib is often used as a non-steroidal antiinflammatory analgesic which prevents the 
synthesis of prostaglandins by inhibiting cyclooxygenase-2 (COX-2). It can effectively relieve pain, but cannot effectively prevent longterm recurrence of the disease [2]. Glucosamine sulfate, a naturally occurring amino sugar in the matrix of cartilage, effectively repairs damaged soft tissue and inhibits the recurrence of inflammation [3]. Previous studies have shown that a combination of celecoxib with glucosamine sulfate could effectively control inflammatory response and reduce its long-term recurrence [4].

The present study was aimed at investigating the combined effect of celecoxib and glucosamine sulfate on inflammatory factors and oxidative stress indicators in patients with KOA.

\section{METHODS}

\section{Patients and general information}

Patients with KOA (120 cases) were recruited over a 1-year period for this study and randomly assigned to two groups of 60 patients each: control group and study group. They consisted of 72 males and 48 females aged 45 to 75 years ( mean age $=60.5 \pm 5.8$ years), with disease duration ranging from 6 months to 15 years. The clinical scores were: mild (64 cases), moderate (40 cases), and severe (19 cases). Their joints activities ranged from 90 to $146^{\circ}$. The inclusion criteria were: (1) patients who met the Guidelines for the Diagnosis and Treatment of Osteoarthritis developed by the Chinese Medical Association [5]; (2) patients aged 40 to 80 years; (3) patients whose X-ray results revealed that the affected joints had mild or obvious osteophyte formation; (4) patients who had normal joint spaces; and (5) patients who signed written informed consent with their family members.

The exclusion criteria were: (1) patients whose joint dysfunctions were caused by other diseases and not rheumatoid arthritis; (2) patients who had severe heart, liver and kidney dysfunctions and systemic diseases; (3) patients who had joint effusion, bony and deformed joints; (4) patients with severe mental or neurological disorders; (5) pregnant and lactating women; and (6) patients who did not comply with the research protocol.

This research received approval from the ethical committee of Department of Sports Medicine, People's Hospital of Ganzhou City, No. 18 Meiguan Avenue, Zhanggong District, Ganzhou City, Jiangxi Province (approval no. 20187887), and was carried out in line with the guidelines of
Helsinki Declaration of 1964 as amended in 1996 [6].

Age, gender, disease duration and clinical symptoms were comparable between both groups (Table 1).

Table 1: Patients' profiles

\begin{tabular}{lcc}
\hline Parameter & Study & Control \\
\hline Gender (male/female) & $35 / 25$ & $37 / 23$ \\
Age (years) & $60.4 \pm 5.6$ & $61.3 \pm 4.9$ \\
Course of disease & $5.6 \pm 1.7$ & $5.2 \pm 1.4$ \\
(years) & 31 & 33 \\
Condition - Mild & 21 & 19 \\
Condition - Moderate & 10 & 9 \\
Condition - Severe & $123.15 \pm$ & $125.26 \pm$ \\
Range of joint activity & 11.04 & 12.11 \\
\hline
\end{tabular}

\section{Treatment regimen}

Control group: Celecoxib was administered at a dose of $200 \mathrm{mg} / \mathrm{kg} /$ day.

Study group: In addition to celecoxib (200 $\mathrm{mg} / \mathrm{kg} /$ day), glucosamine sulfate (500 mg/kg) was administered thrice a day.

Treatment for both groups lasted 8 weeks during which the patients were not allowed to take other anti-osteoarthritic drugs. They were also advised to avoid excessive use of their knee joints, consumption of spicy food, and cold water baths. In addition, they were asked to ensure that they kept themselves warm.

\section{Assessment of efficacy}

Clinical effectiveness was assessed at the end of 8 weeks of treatment and 3 months after, and classified into four grades: healed, remarkably effective, effective and ineffective. Healed: In this group, there was complete disappearance of clinical symptoms, and joint activity was $\leq 135^{\circ}$; remarkably effective: this referred to disappearance of clinical symptoms, absence of pain during walking, occasional pain during activity, and joint activity $\leq 135^{\circ}$; effective: marked by slight improvement in clinical symptoms, mild pain during walking, and limited degree of joint activity; ineffective: here, there were no significant improvements in clinical symptoms. The total effectiveness was calculated as shown in Eq 1.

$\mathrm{TE}(\%)=\{(\mathrm{H}+\mathrm{RE}+\mathrm{E}) / \mathrm{TN}\} \times 100$

where TE is total effectiveness, $\mathrm{H}$ is healed, $\mathrm{RE}$ is remarkably effective, $\mathrm{E}$ is effective and $\mathrm{TN}$ is total number of cases. 


\section{Determination of inflammatory factors}

Joint fluid was taken before and post-treatment, and TNF- $\alpha, I L-1$ and $P E_{2}$ were assayed in serum using ELISA kits.

\section{Evaluation of oxidative status}

Fasting blood (4 $\mathrm{mL}$ ) was drawn from each patient pre- and post-treatment, into plain sample bottles. The serum samples recovered after centrifugation were refrigerated at $-20{ }^{\circ} \mathrm{C}$ and used for assay of serum SOD and level of MDA.

Determination of visual analogue scale (VAS), osteoarthritis index, and Lysholm knee score scale (LKSS)

Pain level was scored using VAS (0 to 10 points). The higher the score, the more severe the pain. The osteoarthritis index was scored according to the Western Ontario and McMaster Universities Osteoarthritis Index (WOMAC). A total of 24 items were used and assigned a total of 96 points. The higher the score, the more severe the degree of OA. LKSS score consisted of eight questions with scores ranging from 0 to 100 . The higher the score, the better the knee function.

\section{Statistical analysis}

Continuous variables following a symmetric distribution are presented as mean \pm standard deviation (SD), and were analyzed with Student's t-test. Numerical data are presented as frequency (\%), and statistical analysis was performed using Chi square. All analyses were carried out with SPSS version 19.0. Statistical significance was fixed at $p<0.05$.

\section{RESULTS}

\section{Treatment effectiveness}

After treatment, the total effectiveness was significantly higher in the observation group $(91.33 \%)$ than in control group $(71.67 \%)(p<$ 0.05). These results are shown in Table 2.

\section{Inflammatory factors}

Before treatment, the levels of TNF- $\alpha, \mathrm{IL}-1$ and $\mathrm{PGE}_{2}$ were similar between patients in both groups $(p>0.05)$. However, post-treatment TNF$\alpha, I L-1$ and $P G E_{2}$ were markedly lower in the glucosamine sulfate-treated patients than in control patients $(p<0.05$; Table 3$)$.

\section{Activity of SOD and level of MDA}

As shown in Table 4, before treatment, SOD activity and MDA level were comparable between patients in both groups $(p>0.05)$. However, after treatment, the activity of SOD was significant higher in patients treated with glucosamine than in control patients $(p<0.05)$. However, there was a marked decrease in MDA level in the glucosamine-treated patients, when compared with control patients $(p<0.05)$.

Table 4: SOD activity and MDA levels $(n=60)$

\begin{tabular}{lccc}
\hline Group & Time point & SOD $(\mathbf{U} / \mathbf{L})$ & $\begin{array}{c}\text { MDA } \\
(\mathbf{n m o l} / \mathbf{m l})\end{array}$ \\
\hline Study & Before & $87.28 \pm$ & $13.25 \pm$ \\
& treatment & 15.32 & 6.39 \\
& After & $103.65 \pm$ & $8.35 \pm$ \\
& treatment & $26.89^{* \#}$ & $5.10^{* \#}$ \\
& Before & $79.36 \pm$ & $16.35 \pm$ \\
Control & treatment & 18.42 & 8.25 \\
& After & $84.56 \pm$ & $14.54 \pm$ \\
& treatment & 37.85 & 7.23 \\
\hline${ }^{*} p<0.05$, when compared to pre-treatment; ${ }^{\#} p<0.05$, \\
relative to control post-treatment
\end{tabular}

Table 2: Therapeutic efficacy ( $n, \%)$

\begin{tabular}{lllllll}
\hline Group & $\mathbf{n}$ & Healed & $\begin{array}{l}\text { Remarkably } \\
\text { effective }\end{array}$ & Effective & Ineffective & $\begin{array}{l}\text { Total } \\
\text { effectiveness }\end{array}$ \\
\hline Observation & 60 & $28(46.67 \%)$ & $22(36.67 \%)$ & $6(10.00 \%)$ & $4(6.67 \%)$ & $93.33 \%^{*}$ \\
Control & 60 & $11(18.33 \%)$ & $12(20.00 \%)$ & $20(33.33 \%)$ & $17(28.33 \%)$ & $71.67 \%$ \\
\hline
\end{tabular}

${ }^{\star} P<0.05$, compared to the control group

Table 3: Levels of inflammatory factors $(n=60)$

\begin{tabular}{lllll}
\hline Group & Time point & TNF- $\boldsymbol{\alpha}$ (ng/L) & IL-1 (ng/L) & PGE $_{\mathbf{2}}$ (ng/L) \\
\hline \multirow{2}{*}{ Study } & Before treatment & $16.59 \pm 4.23$ & $49.20 \pm 9.25$ & $139.54 \pm 18.25$ \\
& After treatment & $12.56 \pm 3.50^{\star \#}$ & $41.25 \pm 7.58^{\star \#}$ & $121.38 \pm 28.68^{\star *}$ \\
\multirow{2}{*}{ Control } & Before treatment & $16.35 \pm 4.20$ & $48.95 \pm 8.36$ & $135.69 \pm 18.21$ \\
& After treatment & $15.28 \pm 3.60$ & $47.51 \pm 7.32$ & $134.64 \pm 17.21$ \\
\hline
\end{tabular}

${ }^{*} P<0.05$, compared to the value before treatment; ${ }^{\#} p<0.05$, compared to control group after treatment 


\section{Joint scores}

After treatment, VAS and WOMAC scores in the two groups were markedly reduced, while the LKSS scores were markedly increased, relative to pre-treatment scores $(p<0.05)$. There were marked decreases in VAS and WOMAC scores, and higher LKSS score in glucosamine-treated patients than in control patients $(p<0.05$; Table 5).

Table 5: VAS, WOMAC and LKSS scores $(n=60)$

\begin{tabular}{|c|c|c|c|c|}
\hline Group & $\begin{array}{l}\text { Time } \\
\text { point }\end{array}$ & VAS & WOMAC & LKSS \\
\hline \multirow{2}{*}{ Study } & $\begin{array}{l}\text { Before } \\
\text { treatment }\end{array}$ & $\begin{array}{c}8.15 \\
\pm \\
1.36\end{array}$ & $\begin{array}{c}75.12 \pm \\
5.49\end{array}$ & $\begin{array}{c}42.36 \pm \\
8.45\end{array}$ \\
\hline & $\begin{array}{l}\text { After } \\
\text { treatment }\end{array}$ & $\begin{array}{c}2.36 \\
\pm \\
0.52^{\star \#}\end{array}$ & $\begin{array}{c}40.35 \pm \\
2.36^{\star \#}\end{array}$ & $\begin{array}{l}87.29 \pm \\
10.38^{\star \#}\end{array}$ \\
\hline \multirow{2}{*}{ Control } & $\begin{array}{l}\text { Before } \\
\text { treatment }\end{array}$ & $\begin{array}{c}8.30 \\
\pm \\
1.25\end{array}$ & $\begin{array}{c}72.56 \pm \\
5.34\end{array}$ & $\begin{array}{c}42.69 \pm \\
8.23\end{array}$ \\
\hline & $\begin{array}{l}\text { After } \\
\text { treatment }\end{array}$ & $\begin{array}{c}5.34 \\
\pm \\
1.01^{*}\end{array}$ & $\begin{array}{c}54.23 \pm \\
3.63^{*}\end{array}$ & $\begin{array}{c}75.63 \pm \\
9.15^{\star}\end{array}$ \\
\hline
\end{tabular}

${ }^{*} P<0.05$, relative to the value pre-treatment; ${ }^{\#} p<$ 0.05 , relative to control post-treatment

\section{Adverse reactions}

During treatment, adverse reactions in the observation group (10.00\%) were significantly lower than those in control group $(21.67 \%, p<$ 0.05). These results are shown in Table 6.

\section{DISCUSSION}

Knee osteoarthritis (KOA), a common form of $\mathrm{OA}$, is caused by degenerative knee disease. Early $O A$ is characterized by painful joints, stiffness, hypertrophy, and limited mobility. In the advanced stage of the disease, loss of joint function is caused by exfoliation of cartilage, necrosis, and bone hyperplasia. This is due to the release of huge amounts of IL-1 and TNF- $\alpha$ in the synovial fluid as a result of phagocytic degradation of synovial cells, which adversely affects the metabolism of chondrocytes [7,8]. Patients with KOA are often treated with nonsteroidal anti-inflammatory drugs and cartilage protective agents because of the trauma associated with and high cost of surgery. Studies have shown that celecoxib and glucosamine sulfate alleviate pain and joint swelling in patients with $\mathrm{KOA}$, and exert control effects on advanced cartilage necrosis [9].

In the present study, total effectiveness was significantly higher in the observation group than in control group. VAS and WOMAC scores were significantly lower in the observation group than in control group. However, the corresponding LKSS score was markedly lower in the control group. Incidents of adverse reactions in the observation group were significantly lower than in control group. These results suggest that celecoxib and glucosamine sulfate may be clinically effective as combined treatment of KOA due to the significant reduction in joint pain and improvement of prognosis. The synergistic effect of the two drugs may stimulate synthesis of glycosaminoglycans, increase viscosity of synovial fluid, improve the lubrication function between the joints, inhibit the progression of $\mathrm{OA}$ and improve joint function [10].

Studies have shown that OA lesions are associated with varying degrees of synovial inflammation and the release of large amounts of inflammatory factors such as TNF- $\alpha$ and IL-1 in the synovial fluid. The TNF- $\alpha$ stimulates chondrocytes to synthesize inflammatory mediators such as IL-1 and PGE 2 . Prostaglandin$2\left(\mathrm{PGE}_{2}\right)$ increases cell permeability, promotes infiltration of inflammatory cells, causes local tissue edema, and aggravates OA. Therefore, TNF- $\alpha$, IL-1 and TNF- $\alpha$ play important roles in the pathogenesis of $\mathrm{OA}[11,12]$.

In the present investigation, after treatment, TNF$\alpha, I L-1$ and $P G E_{2}$ were markedly reduced in the glucosamine sulfate-exposed patients than in control patients. This suggests that the combination of celecoxib and glucosamine sulfate may effectively inhibit the release of inflammatory factors, block the inflammatory response pathway, and alleviate pain in patients with $\mathrm{KOA}$.

Table 6: Comparison of adverse reactions between the two groups $(n=60)$

\begin{tabular}{lcccccc}
\hline Group & $\begin{array}{c}\text { Gastrointestinal } \\
\text { reaction }\end{array}$ & $\begin{array}{c}\text { Joint pain } \\
\text { and swelling }\end{array}$ & $\begin{array}{c}\text { Mild } \\
\text { abdominal } \\
\text { pain }\end{array}$ & Nausea & Constipation & $\begin{array}{c}\text { Total } \\
\text { incidents }\end{array}$ \\
\hline Study & $2(3.33 \%)$ & $2(3.33 \%)$ & $0(0.00 \%)$ & $1(1.67 \%)$ & $1(1.67 \%)$ & $6(10.00 \%)^{*}$ \\
Control & $6(10.00 \%)$ & $2(3.33 \%)$ & $2(3.33 \%)$ & $2(3.33 \%)$ & $1(1.67 \%)$ & $13(21.67 \%)$ \\
\hline
\end{tabular}

$* \frac{C .05, \text { compared to control group }}{P<0}$ 
It is possible that celecoxib exerts antiinflammatory and analgesic influence through suppression of COX-2 which in turn inhibits the release of $P G E_{2}$. Glucosamine sulfate inhibits the activity of alkaline phospholipase $A_{2}$, and promotes the synthesis of collagen in cartilage matrix [13]. These results indicate that the combination of celecoxib and glucosamine sulfate may not only increase anti-inflammatory effects, but may also reduce non-steroidal antiinflammatory drug-induced damage to chondrocytes.

Studies have shown that oxidative stress affects metabolism of collagen in chondrocytes and brings about a decline in normal function of cartilage. As an important antioxidant enzyme, SOD reduces oxidative damage in bone and cartilage of joints by mitigating reactive oxygen species (ROS)-induced cell damage. Malondialdehyde (MDA), one of the most important products of lipid peroxidation, induces apoptosis in chondrocytes, which leads to cartilage atrophy, thereby promoting the development of OA [14]. Glucosamine sulfate treatment led to marked increase in SOD activity, and significant decrease in MDA level. This suggests that the combination of celecoxib with glucosamine sulfate effectively inhibits ROSinduced damage to chondrocytes and delays the degeneration of osteoarticular cartilage. Celecoxib potently inhibits COX-2 activity, reduces the generation of oxygen free radicals, and mitigates osteoarthritis-induced oxidative stress damage [15]. Glucosamine sulfate directly reduces cartilage degradation, inhibits the production of superoxide free radicals, and promotes cartilage regeneration and repair [16]. The combination of the two drugs may reduce chondrocyte damage and strengthen the ability of osteoarthritic patients to resist oxidative stress.

\section{Limitations of the study}

This study investigated the combined effects of celecoxib and glucosamine sulfate on inflammatory factors and oxidative stress indicators in patients with knee osteoarthritis. However, the study lasted only eight weeks. Long-term follow-up of patients after combination therapy was not conducted. Therefore, the longterm side effects of the combination therapy were not determined. These aspects require further studies.

\section{CONCLUSION}

The results obtained in this study demonstrate that the combination of celecoxib and glucosamine sulfate effectively reduces immune inflammatory response, oxidative stress damage and joint pain associated with KOA.

\section{DECLARATIONS}

\section{Conflict of Interest}

No conflict of interest associated with this work.

\section{Contribution of Authors}

We declare that this work was done by the author(s) named in this article and all liabilities pertaining to claims relating to the content of this article will be borne by the authors. All authors read and approved the manuscript for publication. Zeng Guangxuan conceived and designed the study, Deng Gang, Chen Xiaguang, Yang Kanghua, Wu Aiping, Zeng Guangxuan collected and analysed the data, while Deng Gang wrote the manuscript. Deng Gang and Chen Xiaguang contributed equally to this work and should be considered as co-first authors.

\section{REFERENCES}

1. Wu ZJ. The etiology, pathogenesis and treatment progress of knee osteoarthritis. Chin $J$ Med Device 2018; (4): 203-204.

2. Gao J, Gui BJ, Hu KZ, Wang SS. Effect of celecoxib on proliferation of human osteoblasts and its mechanism. Acta Univ Med Anhui 2015; (5): 608-611.

3. Vasiliadis HS, Tsikopoulos K. Glucosamine and chondroitin for the treatment of osteoarthritis. World $\mathrm{J}$ Orthop 2017; 8(1): 608-611.

4. Fu WH, Zhang MX. Therapeutic effect of glucosamine sulfate combined with celecoxib on osteoarthritis. Strait Pharm J 2016; (2): 184-185.

5. Chinese Medical Association Rheumatology Branch. Guide to diagnosis and treatment of osteoarthritis. Chin J Rheumatol 2010; 14(6): 416-419.

6. World Health Organization. Declaration of Helsinki. $\mathrm{Br}$ Med J 1996; 313(7070): 1448-1449.

7. Malfait AM. Osteoarthritis year in review 2015: biology. Osteoarthritis Cartilage 2016; 24(1): 21-26.

8. Weinberger M, Tierney WM, Booher P, Hiner SL. Social support, stress and functional status in patients with osteoarthritis. Social Sci Med 2016; 30(4): 503-508.

9. Weimann $G$, Lubenow $N$, Selleng $K$, Eichler $P$, Albrecht $D$, Greinacher $A$. Glucosamine sulfate does not crossreact with the antibodies of patients with heparin-induced thrombocytopenia. Eur $J$ Haematol 2015; 66(3): 195-199.

10. Liang ZQ, Gu WF, Liao J. Effect of Qingre Jiedu Tongluo Decoction Combined with Celecoxib on Serum Related Inflammatory Factors in Patients with Osteoarthritis. China Med 2018; (3): 433-436. 
11. Yu HJ, Wang CH. Efficacy and safety of glucosamine sulfate in the treatment of knee osteoarthritis. World Latest Med Inf 2015; 15(38): 110-114.

12. Duan DB, Zhang SY. Effect of sodium hyaluronate combined with celecoxib on osteoarthritis and its effect on TNF- $\alpha, I L-1 \beta$ and PGE_2. Med Recapitulate 2016; 22(23): 4729-4732.

13. Zhang L. Clinical analysis of glucosamine sulfate in the treatment of knee osteoarthritis. World Latest Med Inf 2017; (11): 101-102.

14. Ma J, Zuo Z, Ma YS, Pan HZ, Yang WL. A Study on the Relationship between Oxidative Stress Reaction and
Osteoarthritis of Knee. Progress Mod Biomed 2016; 16(3): 500-503.

15. Bruce, Wang CM, Xie XP, Wesley, Furnback, Ronald, Caldwell. Cost-effectiveness study of celecoxib in the treatment of osteoarthritis in China. China Pharm 2017; 28(5): 586-590.

16. Fraioli A, Mennuni G, Fontana M, Nocchi S, Ceccarelli F, Perricone C, Serio A. Efficacy of Spa Therapy, MudPack Therapy, Balneotherapy, and Mud-Bath Therapy in the Management of Knee Osteoarthritis. A Systematic Review. Biomed Res Int 2018; 2018: 1042576. 